\title{
Development of a Post-Processing Automation Procedure for the GPS-Based Travel Time Data Collection Technique
}

\author{
Laura Berzina ${ }^{1}$, Ardeshir Faghri ${ }^{2}$, Morteza Tabatabaie Shourijeh ${ }^{3}$, Mingxin Li $^{2}$ \\ ${ }^{1}$ McCormick Taylor, Inc., Newark, USA \\ ${ }^{2}$ Department of Civil \& Environmental Engineering, University of Delaware, Newark, USA \\ ${ }^{3}$ I.S. Engineers, LLC, Houston, USA \\ Email: lmx@udel.edu
}

Received October 17, 2013; revised November 19; 2013; accepted December 15, 2013

Copyright (C) 2014 Laura Berzina et al. This is an open access article distributed under the Creative Commons Attribution License, which permits unrestricted use, distribution, and reproduction in any medium, provided the original work is properly cited. In accordance of the Creative Commons Attribution License all Copyrights (C) 2014 are reserved for SCIRP and the owner of the intellectual property Laura Berzina et al. All Copyright (C) 2014 are guarded by law and by SCIRP as a guardian.

\section{ABSTRACT}

The travel time data collection method is used to assist the congestion management. The use of traditional sensors (e.g. inductive loops, AVI sensors) or more recent Bluetooth sensors installed on major roads for collecting data is not sufficient because of their limited coverage and expensive costs for installation and maintenance. Application of the Global Positioning Systems (GPS) in travel time and delay data collections is proven to be efficient in terms of accuracy, level of details for the data and required data collection of man-power. While data collection automation is improved by the GPS technique, human errors can easily find their way through the post-processing phase, and therefore data post-processing remains a challenge especially in case of big projects with high amount of data. This paper introduces a stand-alone post-processing tool called GPS Calculator, which provides an easy-to-use environment to carry out data post-processing. This is a Visual Basic application that processes the data files obtained in the field and integrates them into Geographic Information Systems (GIS) for analysis and representation. The results show that this tool obtains similar results to the currently used data post-processing method, reduces the post-processing effort, and also eliminates the need for the second person during the data collection.

\section{KEYWORDS}

Global Positioning Systems (GPS); Post-Processing Tool; VBA; GIS; Kalman Filter

\section{Introduction}

Today's dependence on the automobile has created a downfall in the traffic environment. Peak hour traffic congestion has become an everyday problem throughout the world, and has reduced the quality of our lives. Previous studies reported that accurate measurement of travel time and delay is important for transport planning, land use, public health, selecting and assessing safety countermeasures for various transportation facilities [1-3]. As essential attributes in travelers' route and departure time decision, travel time and delay serve as important quality-of-service measures for dynamic transportation systems [4-7]. This requires new technologies to be developed to study and curtail traffic congestion problems. The travel time data collection method is used to assist the congestion management by documenting congestion information such as travel time, average speed, or delay time
[8]. The use of traditional sensors installed on major roads (e.g. inductive loops, AVI sensors) [9-11] or more recent Bluetooth sensors [12] along arterials and freeways for collecting data is necessary but not sufficient because of their limited coverage and expensive costs for setting up and maintaining the required infrastructure [13]. As a relatively low-cost and high accuracy solution, GPS related data collection techniques have gained acceptance among transportation engineers and practitioners [14]. As a consequence, few software applications have been developed to use the GPS data collection technique in travel time studies.

Automation of the GPS technique is a difficult task; there is no easy way to accomplish the automation of this procedure. Many companies have addressed this problem by developing different applications for collecting and processing travel time data. Jamar Technologies Inc. has developed GPS travel time software that provided op- 
tions to view the summaries of the data. GETT (GPSBased Evaluation of Travel Time), created by the Texas Transportation Institute, has a capability to calculate delay at critical intersections separating the delay reasons: deceleration delay, stopped delay, and acceleration delay. TravTime is the software that is used to process the collected data for the travel studies. This program generates statistics by run and road characteristics, including generation of speed-distance diagrams. Similarly to GETT, this software provides auditing and visualizing capabilities. GPS Leader, by Battle Science and Technology International, is an in-vehicle data collection system that provides travel time study capability by collecting data on vehicle location and speed.

However, most are for industrial use and can be quite complicated. This urged the need to develop the automation application, GPS Calculator, to be simplified and easy-to-use software to conduct post-processing for data collected by using Trimble GPS Receiver and the Aspen software, which obtained the field data.

\section{Current Data Collection Method}

Since 1997, the Delaware Department of Transportation (DelDOT), with help from the Delaware Center for Transportation (DCT) at the University of Delaware, has been using the GPS technique to collect the travel time and delay data on all major roads and highways in the State of Delaware. The resulting data is then integrated into a GIS database (Figure 1(a)) and Google Earth (Figure 1(b)) for the assessment of traffic systems.

GPS data is collected using a Trimble GPS unit with Trimble TerraSync ${ }^{\mathrm{TM}}$ and GPS Pathfinder Office softwares installed. Using the Aspen software, the user creates dictionary files, which contain information about control-points. Additional information can also be entered if necessary. While running the Aspen software, we collect travel time information.

\section{Problems with the Current Data Collection Method}

The field data using Aspen were collected using two people in the test vehicle: a driver and a data collector. The data collector was focusing on the road and its control-points. Each time a control-point location was reached, the person entered a specific key to record the time of the control-point using Aspen, the recorded values become a separate file, called attribute file, while the rest of the GPS points remain in the velocity file.

This approach has two disadvantages. First data collection requires (at least) two people. More importantly, the data collector has the ability to miss control-points, or incorrectly record control-points by either recording them a few seconds too soon or a few seconds too late. Furthermore, data post-processing requires the data re-

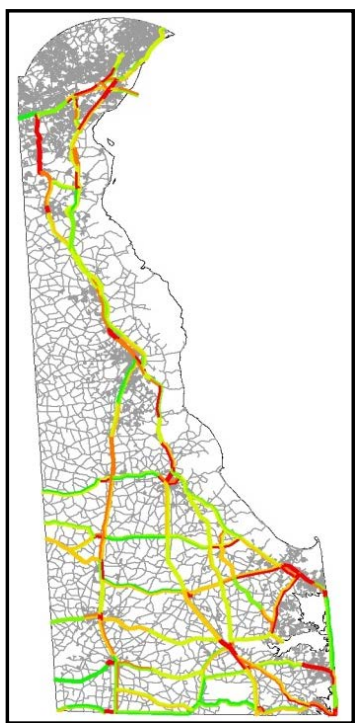

(a)

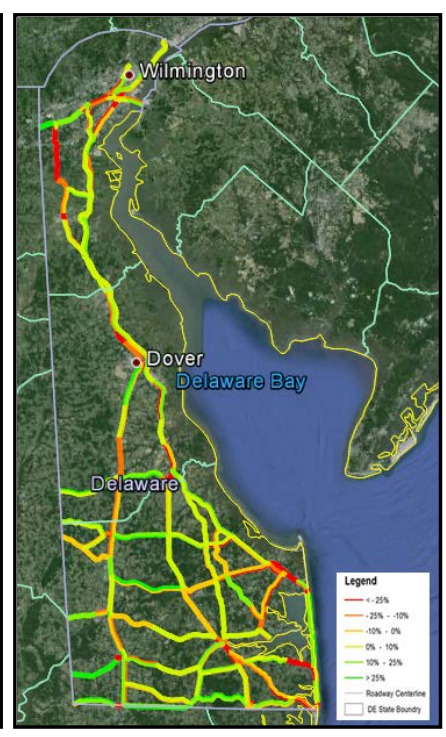

(b)
Figure 1. Sample Travel time and delay measurements in Delaware, USA.

trieval and processing of the data using GPS Pathfinder to obtain the travel time, distance and delay time data. The control-point names had to be fixed after being exported into files if they were missed or pressed wrong. Also, the data files had to follow a certain format for the tool to calculate the distance, travel time, and delay time data. The required work to insure that the data files are in the correct format is time consuming. Moreover, error can be introduced as the data files must be manually check for correspondence between data and controlpoints. This error has the ability to propagate undetected throughout all stages of data post-processing.

\section{Proposed Data Collection and Post-Processing Method}

A new travel data post-processing tool named GPS Calculator, has been created using Visual Basic. This tool provides easy-to-use capabilities and performs the data post-processing task. Previous section described the data collection and post-processing, showing that this is time consuming and error prone. The new software will aim to eliminate some errors from both the collection and postprocessing steps.

First, one person must collect the data by running the Aspen software without recording any data points manually. The only task is to start the software at the beginning of the course run and end it upon finishing. This eliminates one form of human error, being that a user no longer needs to manually record control-points. Secondly, the software can eliminate some error by automating data post-processing.

During the data post-processing, the collected Aspen 
file is exported into the Environmental Systems Research Institute (ESRI) shapefile (.shp) format, which is a data set stored in a spatial format. This format contains time, location, speed and other measures that are used to map the data. It contains the same velocity file data only in a shapefile form. No manual formatting of that data is required. It is important to note that the GPS Calculator is compatible with any file that is in the shapefile format, meaning it is not necessary that the file was created by Aspen.

There are three necessary files needed in order to use the new program:

- the road network shapefile

- the pre-defined control-point information shapefile, and

- the data shapefile we want to process

The road network file contains all the road centerline information for the road network. It is known as a centerline file, because the road lines are based on the center of the road, meaning multiple lanes are represented by a single centerline. Most of the states and countries have such a network created in a spatial format, for example, in a .shp form in ArcGIS or .tab form in Maplnfo.

The control-point shapefile contains all control-points created using the ArcGIS software. For example, if a control-point is at the intersection of Route 273 and Route 58, it is marked on the GIS map at an exact location. The file contains information about each point; by default it has its coordinates only, but additional information could be added, such as the name of the controlpoint, the road it belongs to and so on.

The third file contains the data obtained in the field: location, speed, bearing, time, and other information, recorded in one or two second intervals, depending on satellite signal reception. Occasionally, the intervals could also be longer than a second or two due to multipath.

GPS Calculator uses the obtained data file and checks every single data point entry. First it reads the position expressed in the State Plane Coordinate System, for example, Delaware zone 0700. Bach state has a different state plane zone, with most states having more man one zone. Then it confirms the position with the centerline file, by drawing data points at the obtained location and assigning a corresponding street name for each data point simultaneously, it detects the control-points specified in the control-point shapefile.

\subsection{Mathematical Approach to the Control-Point Search}

The centerline and control-point are detected by drawing a circular radius around each of the data points. The minimum radius of the circle is ten meters (32.81 feet).

The program selects radius using a common formula:

$$
r^{2}=\left(x_{1}-x_{2}\right)^{2}+\left(y_{1}-y_{2}\right)^{2}
$$

The radius corresponds to the average radius of the GPS point according to the width of the road. However, the centerline may not fit perfectly, as the GPS points may be off due to a multipath, or the roads may change over the time, due to construction or some temporary road that is built but does not correspond to the centerline. Therefore, another search can be applied by increasing the GPS point radius. The centerline detection and distance calculation is obtained based on the following trigonometric equations. For visualization of the formulas see Figures 2 and 3.

$$
\begin{aligned}
& \text { adist }=\sqrt{\left(x_{1}-x_{3}\right)^{2}+\left(y_{1}-y_{3}\right)^{2}} \\
& \omega=\tan ^{-1}\left(\frac{y_{2}-y_{1}}{x_{2}-x_{1}}\right) \\
& \alpha=\tan ^{-1}\left(\frac{y_{3}-y_{1}}{x_{3}-x_{1}}\right) \\
& a=\alpha-\omega \\
& \text { pdist }=\operatorname{abs}(\text { adist } \times \sin (a)) \\
& \text { pnt1dist }=\text { adist } \\
& \text { pnt2dist }=\sqrt{\left(x_{2}-x_{3}\right)^{2}+\left(y_{2}-y_{3}\right)^{2}} \\
& \text { ldist }=\sqrt{\left(x_{1}-x_{2}\right)^{2}+\left(y_{1}-y_{2}\right)^{2}} \\
& b a=\sqrt{p_{n t 1 d i s t^{2}-p^{2} \text { dist }^{2}}} \\
& b b=\sqrt{p n t 2 d i s t^{2}-p d i s t^{2}} \\
& c_{1}=\text { ldist }-b a \\
& c_{2}=\text { ldist }-b b
\end{aligned}
$$

where $\left(x_{1}, y_{1}\right)$ is the centerline point, $\left(x_{2}, y_{2}\right)$ is the next centerline point, and $\left(x_{3}, y_{3}\right)$ is the collected GPS point If $c_{1}<0$ or $c_{2}<0$ then it fails, otherwise pdist is used as the true distance. The search for that particular point and segment combination fails because the collected point falls outside of the centerline segment. The program reads all GPS points, and using the formulas given above assigns the closest street's name found in the centerline file.

\subsection{Data Post-Processing with the GPS Calculator}

Using a GPS unit and Aspen software (or other GPS software), one starts to collect the travel data from the start of the course, driving back and forth as many times as needed. Upon completion of the run, one can end the 


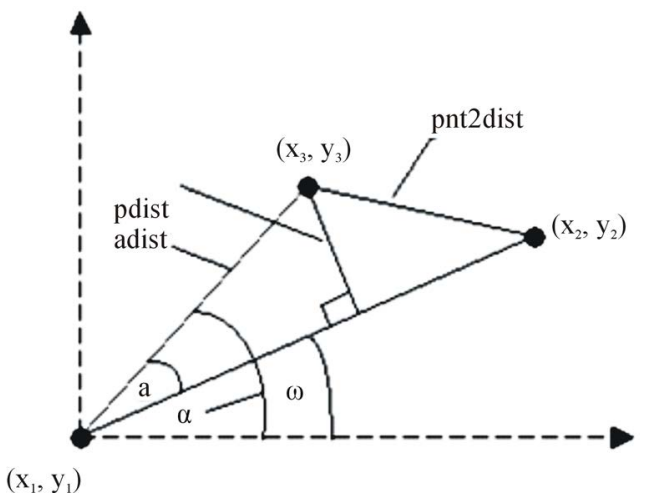

Figure 2. Example of the search algorithm when the collected point falls within the centerline segment.

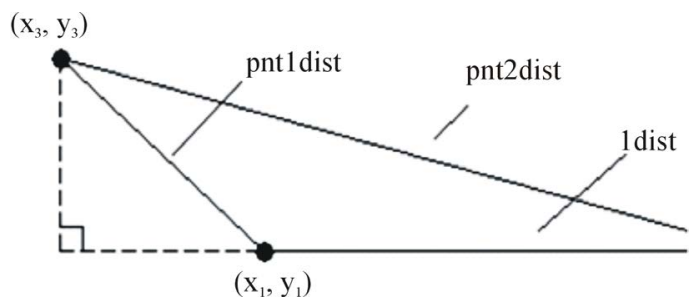

Figure 3. Example of the search algorithm when the collected point falls outside of the centerline segment.

program to stop the run. Next the data is exported into a shapefile format so it is ready to use. The following sections describe using all the functionality of the GPS Calculator.

\subsubsection{Importing Data Files}

After starting GPS Calculator, it is necessary to load the data files into the program. As previously mentioned, three files are required. The user must:

- Choose the road network shapefile

- Choose the control-point shapefile

- Choose the GPS point shapefile

\subsubsection{Importing the Road Network}

To import the road network shapefile, select the Browse button located next to the Road Shape File text box, seen in upper left corner of Figure 4.

Once the file has been selected it must be cached using the Cache button located immediately to the right of the Road Shape File text box. It takes several minutes to process.

However, this procedure only must to be done once, the first time the file is used. Note mat this cache step must be repeated if any changes have been applied to the road network shapefile; the program will always ask you permission.

\subsubsection{Importing the Control-Points}

The user, with the help of ArcGIS 9.0 software, may

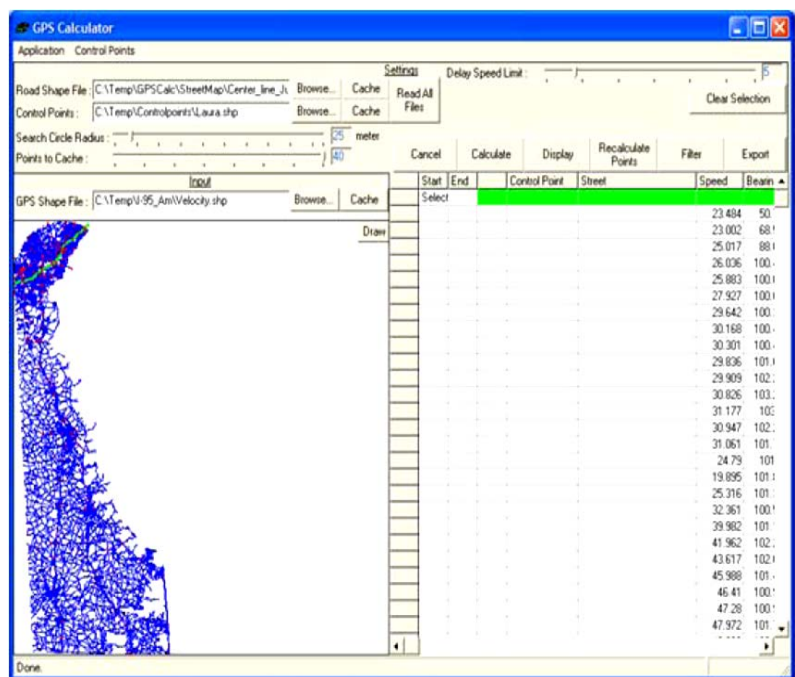

Figure 4. Program interface after selecting necessary shapefiles.

create the control-point file with the location and names of the control-points. For example, intersection of SR 273 and SR 37 can be named "SR 273/SR 37". Inour case, we have created a control-point file, which contains information about control-point names only, using names and numbers of the intersecting roads.

The procedure for importing the control-point shapefile is identical to the road network shapefile importation procedure. Again, click the Browse button located next to the Control Points text box, seen in upper left corner of Figure 4.

Once the file has been selected it must be cached using the Cache button located immediately to the right of the imported file. Again, it takes several minutes to process. However, as before, this procedure must only be done once, the first time the file is used. Note that this cache step must also be repeated if any changes have been applied to the control-point shapefile; the program will always ask you permission.

\subsubsection{Importing the Collected GPS Data}

The third file needed is the actual GPS data file that has been exported from the Aspen software into the shapefile format This file is very important, as it contains the information about the run, including the location of the vehicle every one or two seconds, the speed of the vehicle, the time of the travel, the date of the travel and other values that have been obtained using Aspen field software created by Trimble. This software is no more in production, however, as mentioned before, any data that is in the ESRI shapefile format can be processed with the GPS Calculator.

The procedure for importing the GPS point shapefile is identical to the previous two sections. Again, click the Browse button located next to the GPS Shapefile text 
box, located several fields below the previous shapefile prompts. This can be seen easily in Figure 4. Once thefile has been selected it must be cached using the Cache button located immediately to the right of the imported file. Again, it takes several minutes to process. However, as before, this procedure must only be done once, the first time the .file is used. Note that this cache step must also be repeated if any changes have been applied to the GPS point shapefile; the program will always ask you permission.

\subsubsection{Final Notes on Data Importation}

If the user wishes, it is not necessary to cache the three data files individually. After using Browse, and having valid file names in each associated text box, it is possible to cache all three files simultaneously. This can be done using the Read All Files button. After all three data files are cached; the spreadsheet portion of the interface becomes populated with information from the velocity file. All fields of the table will not be filled, since additional processing is required. This additional processing leads to the next section.

\subsubsection{Calculating GPS Points}

Simply stated, the calculation of the GPS Points is the association of collected GPS data with road names and control-points. This is done using the method discussed in Section 3.1 about mathematical approach. This involves the main processing of the velocity file, which is a time consuming process due to cross-referencing with the other two files and using the search algorithm. In order to calculate GPS points, all the steps about final notes, including caching, should have been completed. After this, simply click the Calculate button, which is located above the spreadsheet portion of the main interface window.

During the calculation process, the current percentage of data that has been processed appears in the bottom right corner of the program interface. When the calculation is completed, the program will display Done in the bottom left corner of the interface. Now the program has identified the control-points that have been found for the default search radius. After clicking Display, the information appears in the fourth column with the heading Control Point

It is possible that all points will be not classified correctly, meaning a point can be associated with an incorrect street name or with an incorrect control-point. Functionality exists to fix these misclassifications; Step 9Street Name Data Filtering handles street name misclassifications and Step 10Recalculating Control-points deals with control-point problems.

\subsubsection{Correction of GPS Measurement Errors}

The smoothing method, Kalman filter, recursively esti- mates outputs using the feedback system. The Kalman filter model can be considered as the combination of two processes: a time updating process and a measurement updating process. The time update and measurement update equations [15] are as follows:

$$
\begin{gathered}
\hat{x}_{k+1}^{-}=A \hat{x}_{k}^{-}+B \mu_{k} \\
P_{k+1}^{-}=A_{k} P_{k} A_{k}^{\mathrm{T}}+Q_{k} \\
K_{k}=P_{k}^{-} H_{k}^{\mathrm{T}}\left(H_{k} P_{k}^{-} H_{k}^{\mathrm{T}}+R_{k}\right)^{-1} \\
\hat{x}_{k}=\hat{x}_{k}^{-}+K_{k}\left(z_{k}-H_{k} \hat{x}_{k}^{-}\right) \\
P_{k}=\left(I-K_{k} H_{k}\right) P_{k}^{-}
\end{gathered}
$$

where

$k=$ time step;

$\hat{x}_{k-1}^{-}=$initial predictor;

$P_{k-1}=$ initial error noise;

$A$ and $B=$ time transition matrices for the prediction process.

$\mu_{k}=$ additional known-input parameter;

$Q=$ prediction error variance;

$\mathrm{K}=$ Kalman gain matrix

$H=$ time transition matrix for the observation process

$Z=$ observed data

$P=$ modified error variance in the Kalman filter

$R=$ measurement error variance.

Because this study uses the second-by-second GPS speed data and tests the Kalman filter separately, the time transition matrix, $A$ is 1 ; and $\mu_{k}$ in Equation (14) becomes zero. The Equations (14)-(18) can be changed into the following form:

$$
\begin{gathered}
\hat{x}_{k+1}^{-}=\hat{x}_{k}^{-} \\
P_{k+1}^{-}=P_{k}+Q_{k} \\
K_{k}=P_{k}^{-}\left(P_{k}^{-}+R_{k}\right)^{-1} \\
\hat{x}_{k}=\hat{x}_{k}^{-}+K_{k}\left(z_{k}-\hat{x}_{k}^{-}\right) \\
P_{k}=\left(I-K_{k}\right) P_{k}^{-}
\end{gathered}
$$

In an example, shown in Figure 5(a), we use a dataset based on measured second-by-second GPS data between 3:05:10PM and 3:23:30PM on 9/26/2012 on the eastbound lanes of State Route 92 Highway, Delaware. As shown in Figure 5(b), since some GPS errors may exist in the data, travel speed can have abrupt changes across the regions. Figure 5(c) shows the results which have been smoothed with Kalman filter to reduce the impacts of noise in the GPS data.

\subsubsection{Visualization of the Data}

After caching the three shapefiles and calculating the 


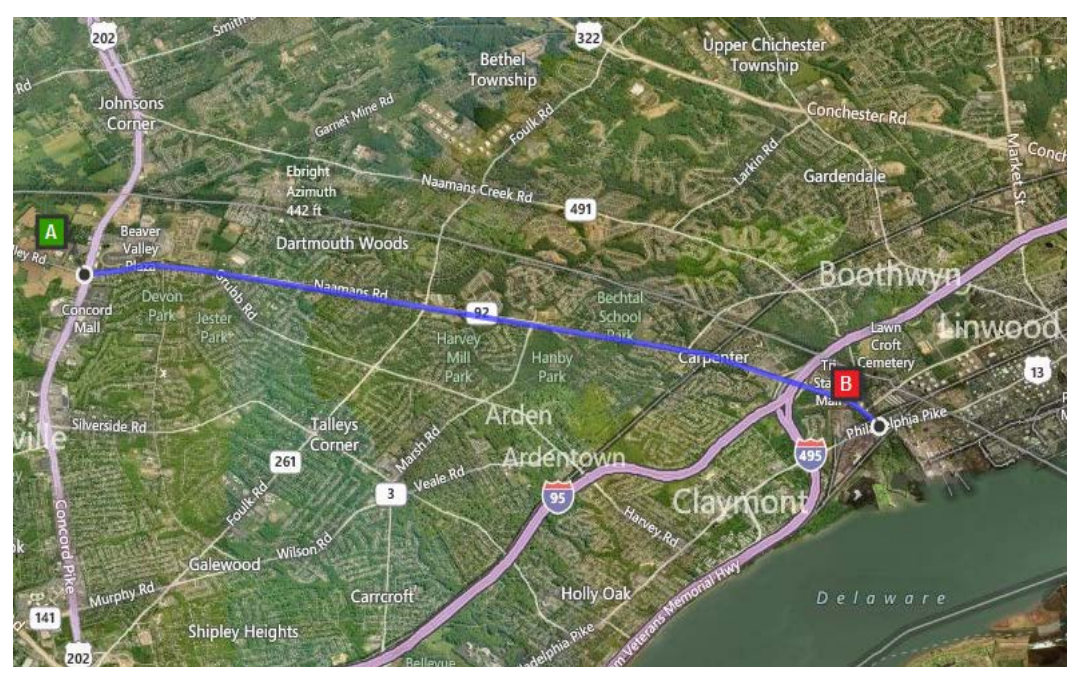

(a)

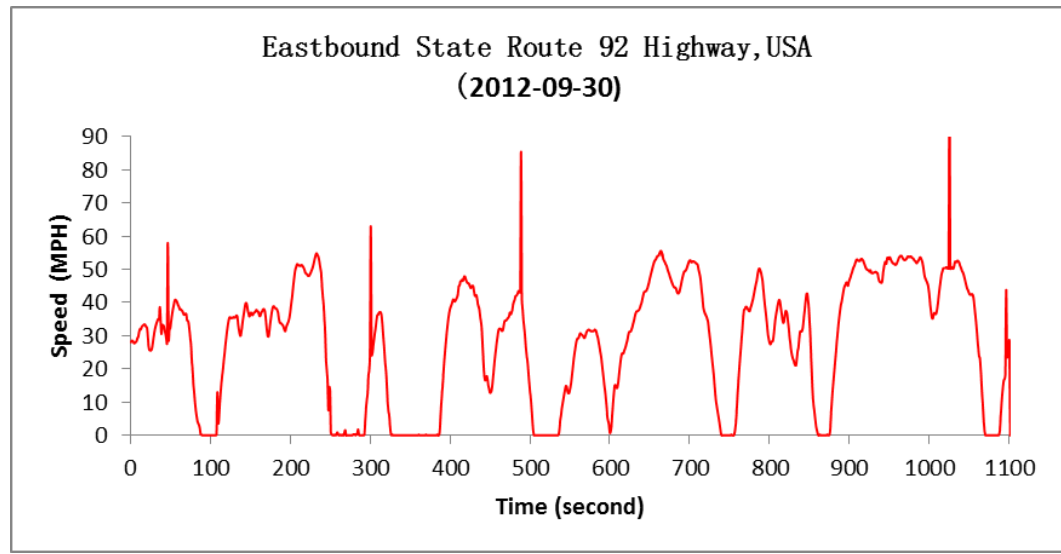

(b)

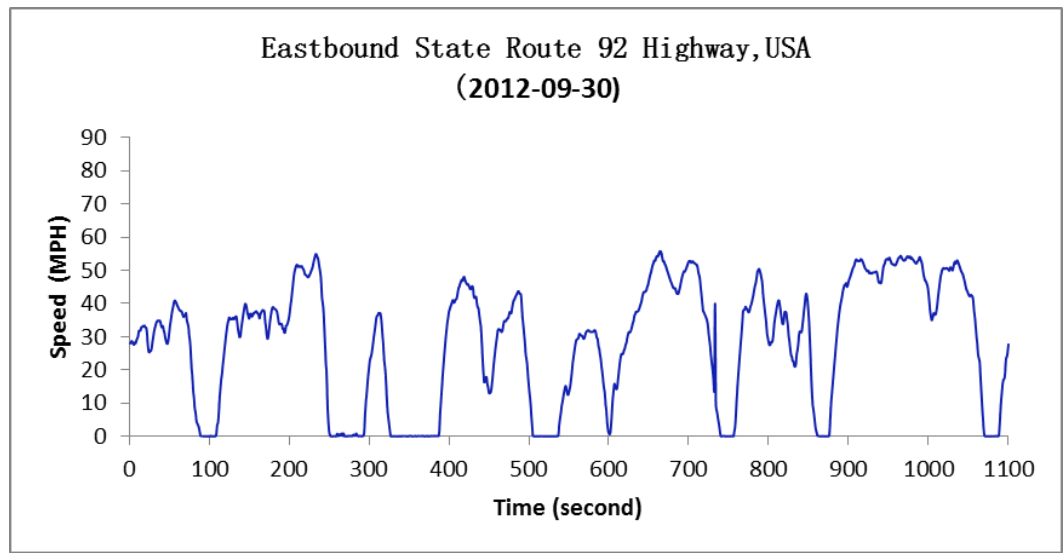

(c)

Figure 5. Correction of GPS measurement errors. (a) Eastbound State Route 92 Highway, Delaware, USA. Source: Bing Maps (2012). (b) Raw GPS data. (c) Smoothed speed with Kalman filter.

GPS points, it is possible to visualize the GPS shapefile. This is done by pressing the Draw button to display the data. The Draw can be seen in Figure 4, immediately below the GPS Shape File Cache button and to the right of the Delaware map. Now one can visualize the spatial location of the current file. When pressing the Draw button, the high level map shows the route location highlighted in green. Furthermore, when pressing any cell in any column to the right of the Control Point column, the map will redraw to a closer view of the selected point. 
This can be seen more clearly in Figure 6. In this example, the intersection of 1-95 and SR 896 is displayed. To return to the high level view, press Draw again.

\subsubsection{Street Name Data Filtering}

An important feature of the GPS Calculator is the ability to correct information. The fifth column, headed Street, contains the street names that the program reads from the centerline file by associating the GPS point to the closest road. As mentioned earlier, it is possible for a GPS point to be misclassified as another road.

Sometimes it detects the side street, as in reality it was the closest point found. However, the program has the capability of filtering these names. Using the Points to Cache slider bar, we can specify how many cells above and below the trouble cell to examine. It checks the street names within this range, and if suddenly mere is a name or two that does not match with the street names given before and after, it filters it out and assigns a new name, that of the surrounding points. This is done by adjusting the slider, and clicking the Filter button.

\subsubsection{Recalculating Control-Points}

As mentioned earlier, it is possible for the GPS Calculator to misidentify control-points. This means it either finds a control-point that does not belong to this route, or more commonly, a control-point does not fall within the search radius. This is common, since most control-points are located at major intersections, and in many cases they are under an overpass, which prevents the GPS signal from being received. Before moving forward, the user must make sure that the correct control-points have been found.

The Search Circle Radius slider bar allows the user to manually control the search radius that the program uses, until all points are found. If the control-point has not been found or is misclassified, click the cell (or cells) in the third column, which correspond to incorrect rows. The cells will turn blue. This can be seen in Figure 7. Resetting the circle radius to a higher value increases the search area for the specified point. To recalculate the selected points, press the Recalculate Points button.

Once again, the status of the calculation will appear in the lower right, and Done will appear in the lower left when the operation is complete. Next press the Display button. The newly found point will replace the incorrect point. This process can be repeated with different radius values until the desired result is reached. At the end of the process unselect all blue cells by using the Clear Selection button, or by clicking on the blue cells.

Generally, this process must be done only once. Once the corrections are made, they will be recorded into the GPS shapefile database; therefore, when opening the file again, the processed information has been saved and will

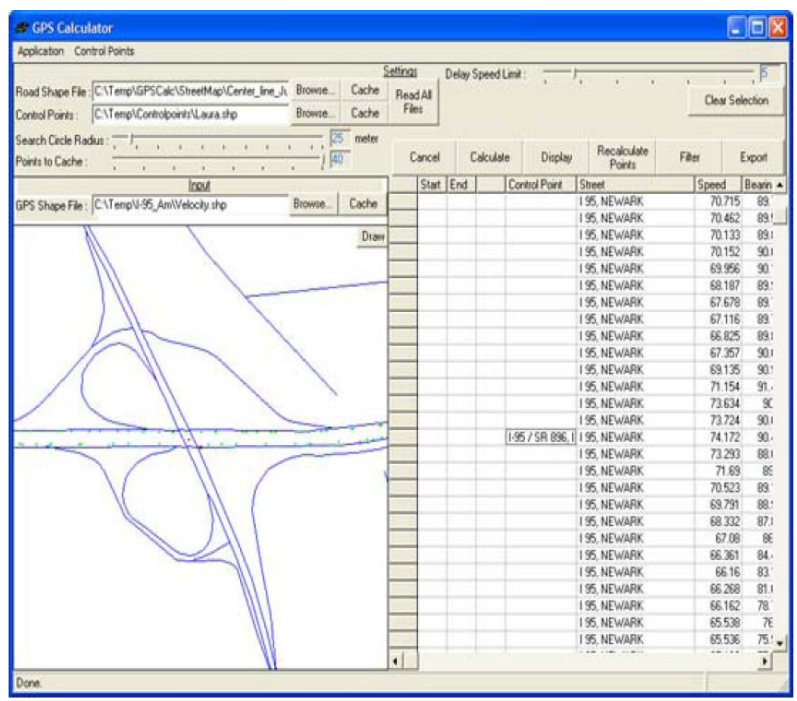

Figure 6. GPS point location by using the corresponding cell.

\begin{tabular}{|c|c|c|c|c|c|c|}
\hline Cancel & \multicolumn{2}{|c|}{ Calculate } & Display & $\begin{array}{l}\text { Recalculate } \\
\text { Points }\end{array}$ & ilter & Export \\
\hline Start & End & & ontrol Point & Street & Speed & Bearin \\
\hline & & & & 1.95, NEW CASTLE & 55.501 & $29 !$ \\
\hline & & & & 1.95, NEW CASTLE & 56.393 & 23. \\
\hline & & & & 1.95, NEW CASTLE & 56.82 & $18 !$ \\
\hline & & & & 1.95, NEW CASTLE & 56.719 & 15.1 \\
\hline & & & $95 / 1-495, \mid-95$ & I.95, NEW CASTLE & 56.309 & 9.1 \\
\hline & & & & 1.95, NEW CASTLE & 56.146 & $3 !$ \\
\hline & & & & 1.95, NEW CASTLE & 55.373 & 0. \\
\hline & & & & 1.95, NEW CASTLE & 55.094 & 357.1 \\
\hline & & & & 1.95, NEW CASTLE & 55.576 & $356 !$ \\
\hline
\end{tabular}

Figure 7. Point selection to recalculate and find controlpoint.

remain there.

\subsubsection{Editing Control-Point Names and Adding} Custom Points

An additional feature provided by GPS Calculator is the capability to delete and change control-point names to different names. These control-point names can be either included names from the control-point shapefile or from a custom list. The first step is to choose the control-point name to be edited. Again, this is done by clicking the cell in the third column, which corresponds to the controlpoint to be changed. It will turn blue. Next the user can right click the highlighted cell. This gives three options: select either, custom control-point; a shapefile controlpoint or clear the control-point All three of these options, along with the cell highlighting, can be seen in Figure 8.

\subsubsection{Custom Points}

If one intends to add a temporary point GPS Calculator provides a feature to add a custom point Click the Con- 


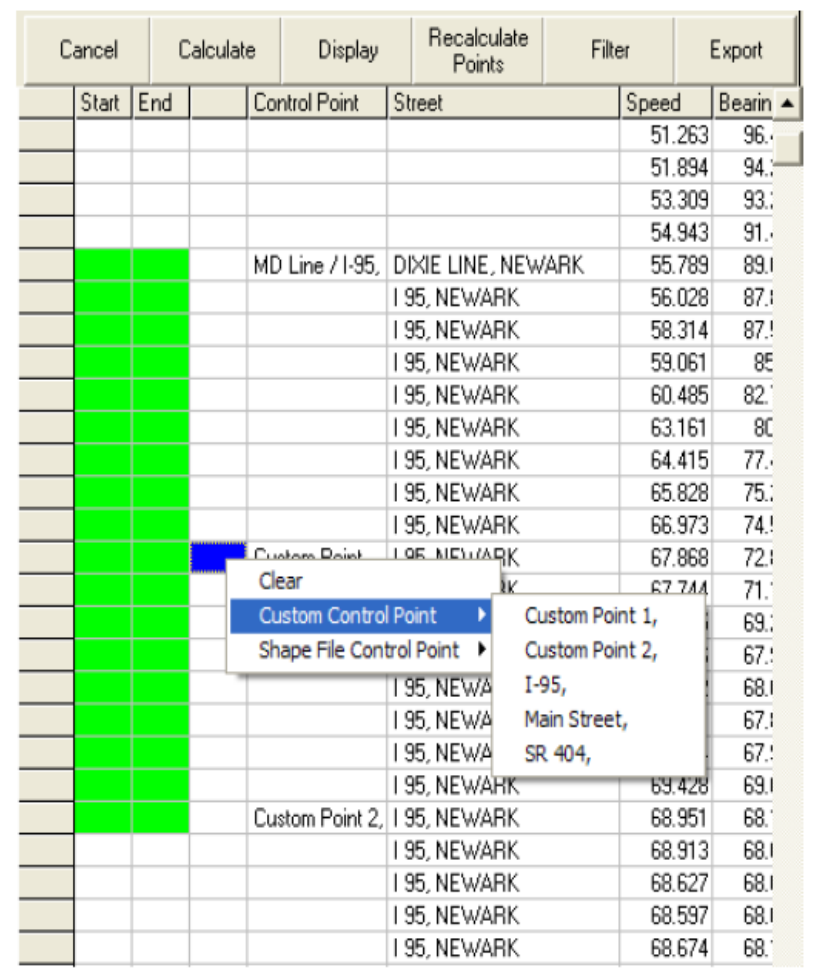

Figure 8. The clear, custom, and shape file options for control-point editing.

trol Point menu at the top of the main interface window. This causes the Control Point dialog box to appear.

Next click the Custom Point Names tab. From here, it is possible to add, remove, and edit the names of custom points. Once they are created, they can be added through cell highlighting and right clicking, as demonstrated in the previous section.

\subsubsection{Selecting and Changing the Desired Beginning and End of the Run}

If the previous steps have been completed successfully, the user can specify which data will be exported by selecting the desired start and end of the run. To select the start of the run, click on the corresponding cell in the first column, Start. To select the end of the run, click the corresponding cell in the second column, End. As shown in Figure 9, the selection turns into green cells. The selection can be edited by once again choosing a different start or different end of the run.

\subsubsection{Data Exporting}

The last step of the data processing is exporting the data into MS Excel data sheets. The data is displayed in Excel and can be saved for later use. The exported file contains these two sheets of data. An important feature for this GFS Calculator is the choice of the delay speed limit The Delay Speed limit bar is located in the upper right comer of the program and offers a range of $1 \mathrm{mph}$ to $30 \mathrm{mph}$.

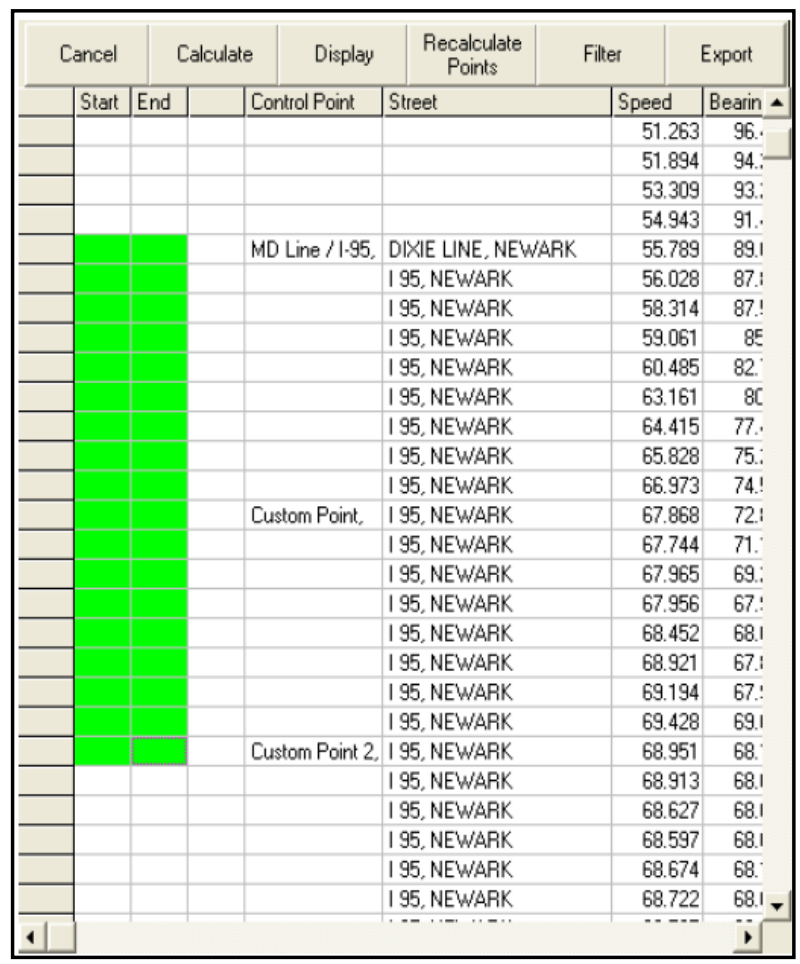

Figure 9. Sample of the beginning and end of the run selection.

This feature is very important as our research group is questioning if the current delay boundary or $5 \mathrm{mph}$ is too low for multilane freeways. Once all of the previous steps are completed, the data can be exported by pressing the Export button. This causes the data to be written into the MS Excel spreadsheet.

The program finds the corresponding attributes to each control-point, i.e., the time when each control-paint was reached. It men computes the delay time between control-points and gives the information output. This information includes distance, travel time, speed, delay, running speed, and the percent of the total time in delay.

\section{Conclusions and Recommendations}

The GPS method has been widely used for travel time studies/acquiring enormous databases with many data points. It is necessary to have a data post-processing tool mat that can help eliminate human error while introducing white collecting and processing the data. The advantage of this GPS method is that it takes advantage of a spatial reference that will always be there. Therefore, using the new program, we can process the same data files over again if the control-points change (moved, deleted or new added).

This allows us to refresh the information and make it comparable with new data if the segments have changed. Since control-point changes apply to the control-point shapefile, it takes only a few minutes to add, delete or 
update the control-point information.

As a result, the GPS Calculator was created to assist with the GPS data post-processing. This tool is better than manually entering control-points, as it eliminates human error while collecting the control-points. The GPS Calculator also introduces features that allow user to create custom control-points and a choice of different speed limits to classify delay. This is very important for congestion management studies, as one is able to use the GPS data files collected in the past and recalculate travel times, speeds, and delay times according to the desired data analysis. Next, we will discuss the advantages and disadvantages of the GPS Calculator.

The future recommendations for the GPS Calculator use include processing of all the collected velocity files. It could be applied to the velocity files that are collected in the past to investigate if the delay criterion of $5 \mathrm{mph}$ is sufficient for freeways, and if not, it could provide new delay thresholds.

\section{Acknowledgements}

This work was partially supported by the Delaware Center for Transportation (DCT) co-sponsored by the University of Delaware and the Delaware Department of Transportation. The authors express their sincere gratitude to Jamar Technologies, Inc., and Paul Phillippi, for installing the car sensor, data collector, and the other equipment to conduct our data studies.

\section{REFERENCES}

[1] L. Zhang, W. Xu and M. Li, "Co-Evolution of Transportation and Land Use: Modeling Historical Dependencies in Land Use and Transportation Decision-Making,” No. OTREC-RR-09-08, 2009.

[2] S. Humphrey, A. Faghri and M. Li, "Health and Transportation: The Dangers and Prevalence of Road Rage within the Transportation System," American Journal of Civil Engineering and Architecture, Vol. 1, No. 6, 2013, pp. 156-163.

[3] R. Frey, A. Faghri and M. Li, "The Development of an Expert System for Effective Countermeasure Identification at Rural Unsignalized Intersections,” International Journal of Information Science and Intelligent System, Vol. 3, No. 1, 2014, pp. 23-40.

[4] M. Li, X. Zhou and N. Rouphail, "Planning-Level Methodology for Evaluating Traveler Information Provision Strategies under Stochastic Capacity Conditions,” Transportation Research Board's, Washington DC, 2011.
[5] M. Li, X. Zhou and N. Rouphail, "Quantifying Benefits of Traffic Information Provision under Stochastic Demand and Capacity Conditions: A Multi-Day Traffic Equilibrium Approach,” 14th International IEEE Conference on Intelligent Transportation Systems Conference (ITSC), Washington DC, 5-7 October 2011, pp. 21182123.

[6] X. Zhou, N. Rouphail and M. Li, “Analytical Models for Quantifying Travel Time Variability Based on Stochastic Capacity and Demand Distributions,” Transportation Research Board 90th Annual Meeting, No. 11-3603, Washington DC, 2011.

[7] A. Jia, X. Zhou, M. Li, N. Rouphail and B. Williams, "Incorporating Stochastic Road Capacity into a Day-to-Day Traffic Simulation and Traveler Learning Framework: Model Development and Case Study," Transportation Research Record: Journal of the Transportation Research Board, Vol. 2254, No. 1, 2011, pp. 112-121. http://dx.doi.org/10.3141/2254-12

[8] H. Mahmassani, R. Mudge, T. Hou and J. Kim, "Use of Mobile Data for Weather-Responsive Traffic Management Models,” US Department of Transportation, 2012.

[9] A. Faghri and K. Hamad, "Application of GPS in Traffic Management Systems,” GPS Solutions, Vol. 5, No. 3, 2002, pp. 52-60. http://dx.doi.org/10.1007/PL00012899

[10] S. Robinson and J. Polak, "Modeling Urban Link Travel Time with Inductive Loop Detector Data by Using the kNN Method," Transportation Research Record: Journal of the Transportation Research Board, Vol. 1935, No. 1, 2005, pp. 47-56. http://dx.doi.org/10.3141/1935-06

[11] F. Dion and H. Rakha, "Estimating Dynamic Roadway Travel Times Using Automatic Vehicle Identification Data for Low Sampling Rates," Transportation Research Part B: Methodological, Vol. 40, No. 9, 2006, pp. 745766. http://dx.doi.org/10.1016/j.trb.2005.10.002

[12] A. Haghani, M. Hamedi, K. Sadabadi, S. Young and P. Tarnoff, "Data Collection of Freeway Travel Time Ground Truth with Bluetooth Sensors," Transportation Research Record: Journal of the Transportation Research Board, Vol. 2160, No. 1, 2010, pp. 60-68.

[13] S. Turner, W. L. Eisele, R. J. Benz and D. J. Holdener, “Travel Time Data Collection Handbook," Federal Highway Administration, Report FHWA-PL-98-035, 1998.

[14] B. Coifman and S. Krishnamurthy, "Vehicle Reidentification and Travel Time Measurement across Freeway Junctions Using the Existing Detector Infrastructure,” Transportation Research Part C: Emerging Technologies, Vol. 15, No. 3, 2007, pp. 135-153. http://dx.doi.org/10.1016/j.trc.2007.03.001

[15] G. Welch and B. Gary, "An Introduction to the Kalman Filter,” 1995. 